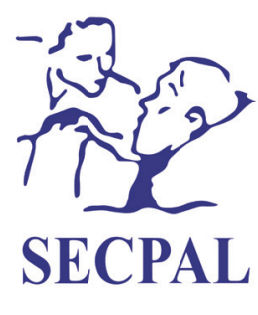

Medicina Paliativa

www.medicinapaliativa.es

ORIGINAL

\title{
Dolor y calidad de vida en pacientes con cáncer de mama
}

\author{
Ricardo Carreño ${ }^{1^{*}}$, Tatiana Vidaurre ${ }^{2}$, Maritza Placencia ${ }^{3}$ y Miguel Otoya ${ }^{4}$
}

Facultad de Medicina, Universidad de San Martín de Porres, Sección de Posgrado-Coordinación de Residentado Médico, Perú. ${ }^{2}$ Instituto Nacional de Enfermedades Neoplásicas, Perú. ${ }^{3}$ Universidad Nacional Mayor de San Marcos, Perú. ${ }^{4}$ Instituto Nacional de Enfermedades Neoplásicas, Perú

Recibido el 18 de junio de 2019

Aceptado el 25 de septiembre de 2020

\section{PALABRAS CLAVE \\ Cáncer de mama, calidad de vida relacionada con la salud, dolor, dolor crónico, cuestionario SF-36, estadio clínico.}

\begin{abstract}
Resumen
Objetivo: Medir la prevalencia de dolor en mujeres con cáncer de mama que han sido tratadas, caracterizar su forma de presentación y valorar la influencia que tiene en la calidad de vida, el dolor y el estadio de cáncer.

Materiales y métodos: Estudio descriptivo, observacional, transversal analítico en 328 pacientes con cáncer de mama del Instituto Nacional de Enfermedades Neoplásicas. Se utilizó la encuesta calidad de vida relacionada con la salud SF-36 y una escala diseñada para medir el dolor.

Resultados: El 87,8 \% tuvo dolor; el 36,1\% dolor severo al cargar objetos, y el 26,4\% al hacer ejercicios. La media de la puntuación total de calidad de vida en todas las pacientes fue $58,2 \pm$ 19,2. Hubo una correlación inversa significativa entre el dolor y la calidad de vida. Las pacientes con cáncer de mama y dolor presentaron afectación de la función física en los diferentes estadios clínicos.

Conclusiones: La mitad de pacientes con cáncer de mama tuvieron dolor moderado a severo. Hubo diferencias estadísticas significativas entre pacientes con y sin dolor para la calidad de vida relacionada con la salud. El estadio clínico 0 tuvo una fuerte correlación con afectación de la función social, y el estadio clínico IV, una fuerte correlación en la dimensión de rol emocional. Pacientes con metástasis estuvieron más afectadas en la función física con baja puntuación del SF-36; de igual modo, en el dolor corporal, vitalidad y salud general.
\end{abstract}

\footnotetext{
*Autor para correspondencia:

Ricardo Aurelio Carreño

Facultad de Medicina, Universidad de San Martín de Porres. Jr. Las Calandrias, n. ${ }^{151-291 . ~ S a n t a ~ A n i t a, ~ L i m a, ~ P e r u ́ ~}$

Correo electrónico: carrenoricardo@yahoo.com
} 


\section{KEYWORDS}

Breast cancer, quality of life, health-related quality of life, pain, chronic pain, SF-36 questionnaire.

\begin{abstract}
Objective: To measure the prevalence of pain in women having undergone treatment for breast cancer, to characterize their presentation, and to assess the influence that pain and cancer stage have on quality of life.

Materials and methods: A descriptive, observational, cross-sectional analytical study in 328 patients with breast cancer from the National Institute of Neoplastic Diseases. The SF-36 HealthRelated Quality of Life questionnaire and a scale designed to measure pain were used.

Results: In all, $87.8 \%$ had pain; severe pain when loading objects, $36.1 \%$ and when exercising, $26.4 \%$. The mean total quality of life score for all patients was $58.2 \pm 19.2$. There was a significant inverse correlation between pain and quality of life. Patients with breast cancer and pain had physical function impairment in different clinical stages.

Conclusions: Half of breast cancer patients had moderate to severe pain. There were significant statistical differences between patients with pain and without pain in health-related quality of life. Clinical stage 0 had a strong correlation with impairment of social function, and clinical stage IV had a strong correlation with the Emotional Role dimension. Patients with metastases had physical function more severely affected with a low SF-36 score; the same was the case with body pain, vitality, and general health.
\end{abstract}

Carreño R, Vidaurre T, Placencia M, Otoya M. Dolor y calidad de vida en pacientes con cáncer de mama. Med Paliat. 2020;27(1):15-23

\section{INTRODUCCIÓN}

El cáncer es un problema de salud pública a escala mundial, pues así lo demuestran sus altas tasas de incidencia y mortalidad. El cáncer de mama afecta seriamente la calidad de vida relacionada con la salud ${ }^{1}$. En Latinoamérica y el Perú, ocupa el tercer lugar de las causas de muerte. En la población general, representa la segunda en morbilidad con $6985(10,5 \%)$ casos anuales después del cáncer de próstata $(11,4 \%)$ y más frecuente que el de estómago $(8,6 \%)^{2}$. El Instituto Nacional de Enfermedades Neoplásicas (INEN) brinda atención a pacientes portadores de tumores malignos, entre ellos el cáncer de mama. Esta es la segunda causa de morbilidad atendida en la mujer peruana y en el INEN; la primera es el cáncer de cuello uterino.

En el Perú, el diagnóstico se ha mejorado con equipamiento y recurso humano en el país a través de la implementación del Plan Esperanza ${ }^{3,4}$ y por el incremento de clínicas y servicios especializados, concentrados mayoritariamente en Lima Metropolitana. Pero existe un insuficiente conocimiento del dolor y la calidad de vida en la mujer peruana con cáncer de mama, lo que se advierte en la ausencia de publicaciones en los últimos 30 años en Perú.

Las características personales de la paciente con cáncer de mama que influyen en la calidad de vida son: edad, procedencia, nivel de educación, religión, expectativas de la paciente, accesibilidad geográfica.

El dolor está presente en la mayoría de las pacientes y afecta su calidad de vida en los diferentes estadios de la enfermedad. El dolor en pacientes con cáncer de mama se presenta porque muchas mujeres experimentan efectos secundarios del tratamiento, incluso dolor crónico (es decir, dolor que dura al menos 3 meses) o se prolonga después de varios años de haber sido diagnosticada y tratada ${ }^{5}$; el dolor, cuando es crónico, puede afectar significativamente la calidad de vida y disminuirla ${ }^{6}$. Aunque la calidad de vida es una calificación subjetiva de la paciente, depende de diferentes condiciones clínicas y personales como: el estadio clínico (EC), la atención recibida y el tratamiento del dolor.

Por otro lado, durante su enfermedad experimenta fatiga o cansancio oncológico, que viene a ser un sentimiento subjetivo y continuo que tiene relación con el tratamiento o el cáncer, e interfiere con la función rutinaria de la mujer con cáncer de mama y es un síntoma frecuente ${ }^{7}$. Se presenta, generalmente, después del sueño o descanso y es de magnitud, regularidad y persistencia superior al cansancio que experimenta una mujer normal, es factor restrictivo para las actividades diarias y tiene una influencia afectiva negativa ${ }^{8}$.

La calidad de vida es un concepto subjetivo, no siempre fácil de mensurar y existen diferentes escalas de medición desarrolladas para cáncer de mama. Una de ellas es el SF-36 ${ }^{9-11}$, que tiene 8 dimensiones y solamente tiene 2 preguntas dentro de la dimensión dolor corporal: si tuvo dolor en alguna parte del cuerpo y hasta qué punto el dolor dificulta el trabajo cotidiano. Lo anterior puede conducir a una caracterización del dolor insuficiente como variable que afecta a la calidad de vida, aunque la encuesta es aceptada y tiene alta confiabilidad para medir la calidad de vida a nivel mundial.

A pesar de que el estadio en que se encuentre la paciente con cáncer de mama influye en la calidad de vida, este no es el único factor, porque depende de la atención oncológi- 
ca recibida, de la capacidad de afrontamiento, del tipo de esquema de tratamiento recibido.

Los EC del cáncer de mama son de 0 a IV. En el estadio 0 el cáncer es local, existe evidencia de células malignas fuera del área de la mama en la que se originó el tumor. El estadio I es denominado cáncer de mama invasivo porque penetra el tejido mamario circundante. En el estadio II no existe masa tumoral mamaria, pero en el examen histopatológico hay células neoplásicas mayores a $2 \mathrm{~mm}$ en $1-3$ ganglios linfáticos axilares o adyacentes al esternón. Además, hay presencia de tumor no mayor a $2 \mathrm{~cm}$ con compromiso de ganglios linfáticos axilares; neoplasia entre $2-5 \mathrm{~cm}$ sin invasión a ganglios linfáticos axilares. En el estadio III hay ausencia de tumor mamario o el tumor puede tener cualquier tamaño, con 4-9 ganglios linfáticos axilares o cercanos al esternón comprometido; la tumoración tiene más de $5 \mathrm{~cm}$, con grupos de células malignas entre $0,3 \mathrm{~mm}$ e inferior a $2 \mathrm{~mm}$ en los ganglios linfáticos; neoplasia mayor a $5 \mathrm{~cm}$ con compromiso de 1-3 ganglios linfáticos axilares o cercanos al esternón; o el tumor de dimensión imprecisa ha tomado la pared torácica o piel mamaria, y ha ocasionado úlcera e inflamación y posible metástasis en 9 ganglios linfáticos axilares o ganglios linfáticos adyacentes al esternón. Hay un tipo de carcinoma inflamatorio de mama, con infiltración de los linfáticos cutáneos mamarios; o la neoplasia está presente en 10 o más ganglios linfáticos axilares, o en ganglios linfáticos subclaviculares, o la neoplasia ha avanzado hacia los ganglios linfáticos axilares o adyacentes al esternón. En el estadio IV el cáncer es invasivo y hay ganglios linfáticos circundantes comprometidos, con metástasis a pulmones, ganglios linfáticos distantes, hígado, huesos y cerebro.

En la atención oncológica integral no se encuentra implementada la medición de calidad de vida, no se prescribe en función de la calidad de vida diagnosticada las interconsultas a otras especialidades, no está insertado en el manual de atención de cáncer de mama el evaluar la calidad de vida; es decir, el abordaje de calidad de vida no está incluido en el tratamiento. Otros factores son las demoras en las citas y las limitaciones en el abastecimiento de medicamentos para pacientes con cáncer de mama resistente (refractario) a fármacos.

Además, existen retos para la mejora de los servicios, porque la información de la calidad de vida no se ha establecido como parte de la atención integral en la atención oncológica, no es un estándar la medición la calidad de vida en las pacientes con cáncer de mama, según tipo de cáncer y EC.

El 20-50 \% de las pacientes se quejan del dolor, y este se incrementa al $90 \%$ para las que tienen metástasis o son pacientes terminales. El dolor es uno de los efectos adversos más frecuentemente reportados que se produce como parte del proceso de la enfermedad o como un efecto secundario de tratamiento ${ }^{12,13}$. Es un problema para la mayoría de las pacientes con cáncer de mama y tiene un efecto desfavorable en la calidad de vida. Según un metanálisis, el dolor prevalece en el $39,3 \%$ de los casos después de tratamiento curativo; en el $55 \%$ durante el tratamiento del cáncer, y en el $66,4 \%$ en estadios avanzados o terminales de la enfermedad $^{14}$.

El objetivo del estudio fue describir el dolor en las mujeres con cáncer de mama, sus características personales y la calidad de vida, según el estadio de cáncer.

\section{MATERIALES Y MÉTODOS}

El estudio de investigación fue de tipo descriptivo, observacional, transversal analítico en pacientes con cáncer de mama. Para el cálculo de tamaño de muestra, se tuvo como referencia al total de atendidos al año por cáncer de mama en el INEN; con una frecuencia esperada del $50 \%$, con un margen de error del $5 \%$. El tamaño calculado fue 319 pacientes con cáncer de mama y la muestra final de pacientes para el estudio fue 328. Los casos fueron obtenidos de los registros del INEN de Perú y se recogieron datos entre los años 2017 y 2018.

Las variables del estudio fueron clasificadas en: personales, sociodemográficas, clínicas, de tipo de atención, de dolor y calidad de vida. En las variables personales se consideró la edad; como variables sociodemográficas el estado civil, el grado de instrucción, la etnia, la religión y la procedencia. Como variables clínicas, la fatiga, las metástasis y el EC; como variable de tipo de atención fue según financiamiento. Las variables de dolor fueron: dolor en $24 \mathrm{~h}$, días con dolor en el mes, grado de dolor, dolor al movilizar el brazo, dolor al agarrar objetos, dolor en la noche cuando se encuentra en cama, dolor cuando descansa en el día, dolor al cargar objetos, dolor al hacer ejercicios, dolor en el trabajo habitual, dolor en las actividades del hogar y rigidez del brazo al final del día.

Se recibió la aprobación ética del Comité Revisor de Protocolos del Departamento de Investigación del INEN para el estudio (carta n. ${ }^{\circ}$ 056-2017-CRP-DI-DICON/INEN). Antes de iniciar la entrevista, se explicó la finalidad del estudio, y para el piloto las pacientes que participaron firmaron el consentimiento informado. Una vez validada la encuesta, se pidió a cada paciente con cáncer de mama firmar un formulario de consentimiento libre e informado. Esto aseguró que la participación fuese voluntaria y que las respuestas se mantendrían anónimas y de manera confidencial.

\section{Instrumento de medición}

Respecto a la escala de medición de la calidad de vida relacionada con la salud, se utilizó el SF-36 $6^{15}$, útil y confiable para cáncer de mama ${ }^{16}$ y se diseñó (adoptó) una escala de medición para el dolor en este tipo de paciente.

De la escala para medir la calidad de vida relacionada a la salud: se utilizó el SF-36, que tiene en su estructura 8 dimensiones: salud general, función física, rol físico, función social, dolor corporal, vitalidad, rol emocional y salud mental. Tuvo validación lingüística y con un test de fiabilidad de 0,894 (alfa de Cronbach). La encuesta se realizó en un promedio de $15 \mathrm{~min}$. Para cada pregunta, las respuestas a cada una de ellas se codificaron a una escala de 0 (peor salud) a 100 (mejor salud). En la población de estudio, las dimensiones de la escala SF-36 fueron fiables y demostraron buena consistencia interna: salud general (alfa de Cronbach: 0,645), función física (alfa de Cronbach: 0,871), rol físico (alfa de Cronbach: 0,871), función social (alfa de Cronbach: 0,661 ), dolor corporal (alfa de Cronbach: 0,748 ), vitalidad (alfa de Cronbach: 0,769), rol emocional (alfa de Cronbach: 0,871 ) y salud mental (alfa de Cronbach: 0,817 ).

De la escala para medir el dolor ${ }^{17}$ : se diseñó un instrumento práctico de tipo Likert para medir el dolor en consulta externa de pacientes con cáncer de mama para Lima Metropolitana (escala Lima), que fue validada por profesionales 
médicos antes de realizar el estudio. Esta escala se realizó para complementar los conocimientos sobre el dolor en pacientes con cáncer de mama del INEN. Especifica el dolor en el ambiente habitual, relacionado con sus actividades diarias, al finalizar el día y al momento de dormir. Los criterios para validarla fueron: consulta a expertos, utilización de criterios de Moriyama y test de fiabilidad. En la consulta a expertos participaron 2 mastólogos y 3 médicos especialistas en tratamiento paliativo. Se les presentó la escala con preguntas tipo Likert, con 10 preguntas: grado de dolor, dolor al movilizar el brazo, dolor al agarrar objetos, dolor en la noche cuando se encuentra en cama, dolor cuando descansa en el día, dolor al cargar objetos, dolor al hacer ejercicios, dolor en el trabajo habitual, dolor en las actividades del hogar y rigidez del brazo al final del día. Cada pregunta tuvo una puntuación de 0 (ausencia de dolor) a 5 (demasiado dolor). La puntuación 1 y 2 corresponden a dolor leve, 3 a dolor moderado, 4 y 5 a dolor severo. La encuesta tuvo un análisis de fiabilidad de alfa de Cronbach de 0,931.

Los criterios de inclusión fueron pacientes adultas de 18 años o mayores, con diagnóstico de cáncer de mama atendidas en el Instituto Nacional de Enfermedades, mujeres con cáncer de mama en EC 0, I, II, III y IV; pacientes que acudan al servicio de medicina y/o cirugía. Pacientes con un mes de enfermedad como mínimo. Los criterios de exclusión fueron: mujeres con cáncer mamario sin estadio al diagnóstico, cáncer de mama bilateral y pacientes tratadas que no deseen participar del estudio.

De la recolección y control de calidad de la data colectada: se diseñó la encuesta en Epi Info 7,2, fue colectada con Epi Info Mobile y Epi Info Cloud. Analizada con Stata 14.

Se realizó un análisis univariado, presentando tablas con frecuencias y porcentajes. En la verificación de hipótesis, si existen diferencias entre los estadios de calidad de vida según dimensiones se utilizó la prueba de Kruskal-Wallis para las dimensiones que cumplen criterios no paramétricos. Se utilizó ANOVA para evaluar los tratamientos y la prueba post hoc Scheffé y análisis de correlación para el dolor.

\section{RESULTADOS}

\section{Características personales de la paciente con cáncer de mama}

La media de la edad fue 52,8 años y con una desviación estándar de 11,7 años.
Los grupos etarios más frecuente con cáncer de mama fueron entre 40-50 años (31,7\% [104]), 50-60 años (29,6\% [97]) y 60-70 años (18,0\% [59]). Predomina el estado civil casada con un $50,9 \%(167)$, el grado de instrucción secundaria con un $47,9 \%$ (157), la etnia mestiza con un 92,1\% (302), la religión católica con un 77,7 \% (255), el estadio de cáncer de mama tipo II con un 44,5 \% (146) y el tipo de atención financiado por el Fondo Intangible Solidario de Salud (FISSAL) con un $65,5 \%$ (215).

La media de la edad de pacientes con cáncer de mama fue 52,9 años, con una mediana de 52 años, un rango de edades entre 23-88 años. La media de enfermedad en meses fue 43,1; la media de días con dolor en el mes fue 19 días. La media de dolor por día fue $5,4 \mathrm{~h}$ y la mediana $28,5 \mathrm{~h}$ (Tabla I).

El $80,9 \%$ de pacientes atendidas procede de 10 regiones: Lima el 34,8 \% (114), Piura el 9,1 \% (30), Ancash el 7,6 \% (25), Lambayeque el 5,2 \% (17), Junín el 4,9\% (16), Cajamarca el 4,6\% (15), Lima provincia el 4,3\% (14), Ica el $4 \%(13)$, Callao el 3,4\% (11) y Cuzco el $3 \%$ (10).

\section{Del dolor en las mujeres con cáncer de mama}

No se encontraron diferencias estadísticamente significativas respecto a la edad en los grupos o EC ( $p$-valor Chicuadrado bilateral 0,697 ), tampoco según etnias ( $p$-valor Chi-cuadrado bilateral 0,264 ), grado de instrucción ( $p$-valor Chi-cuadrado bilateral 0,505$)$, religión ( $p$-valor Chi-cuadrado bilateral 0,754$)$, por lo cual los grupos son homogéneos respecto a estas variables. Se encontraron diferencias en los grupos de EC con el tipo de atención con el grupo FISSAL: seguro subsidiado, comparado con los otros grupos EsSalud, seguro contributivo y hospitalar, los que pagan su consulta ( $\mathrm{p}$-valor Chi-cuadrado bilateral 0,047).

De 328 pacientes con cáncer de mama, 288 (87,8\%) tuvieron dolor. Según el grado de dolor calificado, tuvieron dolor severo el 23,3\%. Según los diferentes tipos de presentación de dolor severo fueron: al movilizar el brazo el 25,7 \%, al agarrar objetos el $25,7 \%$, cuando se encuentra en cama el $24 \%$, al descansar en el día el $160 \%$, al cargar objetos el 36,1\%, al hacer ejercicios el 26,4\%, al trabajo habitual el $21,2 \%$, al realizar actividades del hogar el $22,9 \%$, y rigidez con del brazo al final del día el 21,6 \% (Tabla II).

La media de la puntuación total de calidad de vida en todas las pacientes fue $58,2 \pm 19,2$. Se encontraron diferencias estadísticamente significativas entre las medias de la puntuación

Tabla I. Medidas de tendencia central y de resumen en pacientes con cáncer de mama

\begin{tabular}{|c|c|c|c|c|c|c|c|c|c|}
\hline & \multirow{2}{*}{ Media } & \multirow{2}{*}{$\begin{array}{l}\text { Desviación } \\
\text { estándar }\end{array}$} & \multirow{2}{*}{ Varianza } & \multirow{2}{*}{ Mínimo } & \multirow{2}{*}{ Máximo } & \multicolumn{3}{|c|}{ Percentiles } & \multirow{2}{*}{ Moda } \\
\hline & & & & & & 25 & 50 & 75 & \\
\hline Edad (años) & 52,9 & 11,7 & 136,9 & 23 & 88 & 45 & 52 & 61 & 47 \\
\hline $\begin{array}{l}\text { Tiempo enfermedad } \\
\text { (meses) }\end{array}$ & 43,1 & 58,7 & 3441,3 & 1 & 442 & 8 & 18 & 52 & 12 \\
\hline Dolor en $24 \mathrm{~h}$ & 5,4 & 6,8 & 45,8 & 0 & 24 & 1 & 3 & 6,8 & 1 \\
\hline Días con dolor en el mes & 19 & 12,5 & 155,7 & 0 & 30 & 5 & 28,5 & 30 & 30 \\
\hline
\end{tabular}


Tabla II. Características del dolor en pacientes con cáncer de mama

\begin{tabular}{|c|c|c|c|c|c|c|c|c|}
\hline & \multicolumn{8}{|c|}{ Dolor } \\
\hline & \multicolumn{2}{|c|}{ No dolor } & \multicolumn{2}{|c|}{ Leve } & \multicolumn{2}{|c|}{ Moderado } & \multicolumn{2}{|c|}{ Severo } \\
\hline & $\mathrm{n}$ & $\%$ & $\mathrm{n}$ & $\%$ & $\mathrm{n}$ & $\%$ & $\mathrm{n}$ & $\%$ \\
\hline Grado de dolor & 31 & 10,8 & 104 & 36,1 & 86 & 29,9 & 67 & 23,3 \\
\hline Dolor al movilizar el brazo & 84 & 29,2 & 76 & 26,4 & 54 & 18,8 & 74 & 25,7 \\
\hline Dolor al agarrar objetos & 108 & 37,5 & 64 & 22,3 & 42 & 14,6 & 74 & 25,7 \\
\hline Dolor en la noche cuando se encuentra en cama & 97 & 33,7 & 74 & 25,7 & 48 & 16,7 & 69 & 24 \\
\hline Dolor cuando descansa en el día & 119 & 41,3 & 85 & 29,5 & 38 & 13,2 & 46 & 16 \\
\hline Dolor al cargar objetos & 58 & 20,1 & 70 & 24,3 & 56 & 19,4 & 104 & 36,1 \\
\hline Dolor al hacer ejercicios & 102 & 35,4 & 72 & 25 & 38 & 13,2 & 76 & 26,4 \\
\hline Dolor en el trabajo habitual & 107 & 37,2 & 77 & 26,7 & 43 & 14,9 & 61 & 21,2 \\
\hline Dolor en las actividades del hogar & 92 & 31,9 & 92 & 32 & 38 & 13,2 & 66 & 22,9 \\
\hline Rigidez del brazo al final del día & 85 & 29,5 & 91 & 31,6 & 50 & 17,4 & 62 & 21,6 \\
\hline
\end{tabular}

$n=288$ pacientes con dolor. Fuente: Enien y cols ${ }^{1}$.

global de la calidad de vida (SF-36) en las pacientes con cáncer de mama según estadio, al comparar los grupos con y sin dolor: 1) Estadio 0 con dolor $53,7 \pm 20,5$ y sin dolor $75,4 \pm 0$ (p: 0,000$), 2$ ) Estadio I con dolor $57,6 \pm 19,2$ y sin dolor $70,5 \pm 12,7$ (p: 0,000), 3) Estadio II con dolor 58,1 $\pm 18,1$ y sin dolor $69,3 \pm 10,8$ (p: 0,000), 4) Estadio III con dolor $48,7 \pm 18,6$ y sin dolor $81,1 \pm 0$ (p: 0,000), 5) Estadio IV con dolor $55,4 \pm 18,4$ y sin dolor $82,5 \pm 12,9$ (p: 0,000$)$. Se encontró una correlación inversa entre el dolor y la puntuación total de la Escala de Calidad de Vida Relacionada con la Salud SF-36 ( $r=-0,257$; p: 0,000).

Asimismo, el dolor en pacientes que se encuentran acostumbradas a vivir con dolor fue del 28,8 \% (83), dolor esporádico $10,8 \%(31)$, el intervalo de horas de dolor en la mama o brazo más frecuente fue $2-6$ h (43,8 \% [126]) por día, el intervalo de días con dolor más frecuente al mes fue de 21-30 días (62,5\% [180]) (Tabla III).

\section{Calidad de vida según el estadio clínico en pacientes con cáncer de mama}

Todas las pacientes presentan afectación en las 8 dimensiones de la calidad de vida. El promedio de la puntuación global y desviación estándar de la calidad de vida de pacientes con cáncer de mama fue 58,2 \pm 19,2; para el EC 0 66,6 \pm 18,9; EC I 53,9 \pm 17,7; EC II 60,2 \pm 18,9; EC III 56,4 4 19,5; EC IV 53,4 $\pm 18,3$.

Al análisis bivariado de las características del dolor, las pacientes con cáncer de mama presentaron diferencias significativas en la dimensión función física entre los diferentes EC (p: 0,017, prueba de Kruskal-Wallis) (Tabla IV).

La fatiga está moderadamente relacionada con la función física, rol físico, dolor corporal, vitalidad y salud mental (Tabla V).

El EC 0 (cáncer de mama local) presentó una fuerte correlación $(0,545)$ con afectación de la función social, disminución del esmero en el trabajo y mayor interferencia en horas
Tabla III. Características del dolor en pacientes con cáncer de mama

\begin{tabular}{|c|c|c|c|}
\hline & & $\mathrm{n}$ & $\%$ \\
\hline \multirow{3}{*}{$\begin{array}{l}\text { Acostumbrada a vivir con } \\
\text { dolor }\end{array}$} & Sí & 83 & 28,8 \\
\hline & No & 205 & 71,2 \\
\hline & Total & 288 & 100 \\
\hline \multirow{3}{*}{$\begin{array}{l}\text { Dolor esporádico } \\
\text { (de vez en cuando) }\end{array}$} & Sí & 31 & 10,8 \\
\hline & No & 257 & 89,2 \\
\hline & Total & 288 & 100 \\
\hline \multirow{6}{*}{$\begin{array}{l}\text { Horas de dolor en la } \\
\text { región de la mama o } \\
\text { brazo en } 24 \mathrm{~h}\end{array}$} & Hasta $1 \mathrm{~h}$ & 80 & 27,8 \\
\hline & $2-6 \mathrm{~h}$ & 126 & 43,8 \\
\hline & $6-12 \mathrm{~h}$ & 39 & 13,5 \\
\hline & $12-18 \mathrm{~h}$ & 14 & 4,9 \\
\hline & $18-24 \mathrm{~h}$ & 29 & 10,1 \\
\hline & Total & 288 & 100 \\
\hline \multirow{5}{*}{ Días con dolor } & 1-7 días & 62 & 21,5 \\
\hline & 7-14 días & 14 & 4,9 \\
\hline & 14-21 días & 32 & 11,1 \\
\hline & 21-30 días & 180 & 62,5 \\
\hline & Total & 288 & 100 \\
\hline \multirow{6}{*}{$\begin{array}{l}\text { Horas de dolor en la } \\
\text { región de la mama } \\
\text { o brazo en } 24 \mathrm{~h}\end{array}$} & Hasta 1 hora & 80 & 27,8 \\
\hline & $2-6 \mathrm{~h}$ & 126 & 43,8 \\
\hline & $6-12 \mathrm{~h}$ & 39 & 13,5 \\
\hline & $12-18 \mathrm{~h}$ & 14 & 4,9 \\
\hline & $18-24 \mathrm{~h}$ & 29 & 10,1 \\
\hline & Total & 288 & 100 \\
\hline \multirow{5}{*}{ Días con dolor } & 1-7 días & 62 & 21,5 \\
\hline & 7-14 días & 14 & 4,9 \\
\hline & 14-21 días & 32 & 11,1 \\
\hline & 21-30 días & 180 & 62,5 \\
\hline & Total & 288 & 100 \\
\hline
\end{tabular}

$n=288$ pacientes con dolor. Fuente: International Agency for Research Cancer ${ }^{2}$. 
Tabla IV. Calidad de vida en pacientes con cáncer de mama. Prueba de Kruskal-Wallis

\begin{tabular}{|c|c|c|c|c|c|c|c|}
\hline Dimensión & Estadio & $\mathrm{n}$ & Media & $\begin{array}{l}\text { Desviación } \\
\text { estándar }\end{array}$ & Mínimo & Máximo & Prueba de Kruskal-Wallis \\
\hline \multirow{5}{*}{ Salud general } & 0 & 15 & 63,333 & 20,4397 & 18,8 & 93,8 & \multirow{5}{*}{0,536} \\
\hline & I & 19 & 56,250 & 20,4124 & 25 & 100 & \\
\hline & II & 146 & 62,414 & 19,5033 & 12,5 & 100 & \\
\hline & III & 128 & 58,984 & 20,2827 & 0 & 100 & \\
\hline & IV & 20 & 61,250 & 26,2516 & 18,8 & 100 & \\
\hline \multirow{5}{*}{ Función física } & 0 & 15 & 74,333 & 23,2891 & 15 & 100 & \multirow{5}{*}{0,017} \\
\hline & I & 19 & 59,211 & 30,3344 & 0 & 100 & \\
\hline & II & 146 & 66,130 & 25,3987 & 0 & 100 & \\
\hline & III & 128 & 58,946 & 23,3546 & 0 & 100 & \\
\hline & IV & 20 & 56,000 & 26,6853 & 15 & 100 & \\
\hline \multirow{5}{*}{ Rol físico } & 0 & 15 & 51,667 & 41,6905 & 0 & 100 & \multirow{5}{*}{0,191} \\
\hline & I & 19 & 42,105 & 41,7105 & 0 & 100 & \\
\hline & II & 146 & 39,726 & 42,9185 & 0 & 100 & \\
\hline & III & 128 & 34,375 & 39,6838 & 0 & 100 & \\
\hline & IV & 20 & 20,000 & 29,9122 & 0 & 100 & \\
\hline \multirow{5}{*}{ Función social } & 0 & 15 & 73,333 & 20,5215 & 25 & 100 & \multirow{5}{*}{0,168} \\
\hline & 1 & 19 & 55,263 & 20,5463 & 12,5 & 87,5 & \\
\hline & II & 146 & 63,613 & 26,1758 & 0 & 100 & \\
\hline & III & 128 & 60,938 & 24,5029 & 12,5 & 100 & \\
\hline & IV & 20 & 66,250 & 23,3326 & 37,5 & 100 & \\
\hline \multirow{5}{*}{ Dolor corporal } & 0 & 15 & 71,167 & 28,0762 & 10 & 100 & \multirow{5}{*}{0,119} \\
\hline & I & 19 & 53,947 & 24,8092 & 10 & 100 & \\
\hline & II & 146 & 59,743 & 24,9771 & 10 & 100 & \\
\hline & III & 128 & 56,992 & 25,4456 & 10 & 100 & \\
\hline & IV & 20 & 50,125 & 26,4261 & 10 & 100 & \\
\hline \multirow{5}{*}{ Vitalidad } & 0 & 15 & 68,333 & 25,1819 & 28,3 & 100 & \multirow{5}{*}{0,267} \\
\hline & 1 & 19 & 57,544 & 20,4982 & 8,3 & 93,3 & \\
\hline & II & 146 & 64,224 & 22,2664 & 0 & 100 & \\
\hline & III & 128 & 60,951 & 22,6857 & 15 & 100 & \\
\hline & IV & 20 & 68,500 & 24,4405 & 30 & 100 & \\
\hline \multirow{5}{*}{ Rol emocional } & 0 & 15 & 60,000 & 47,4760 & 0 & 100 & \multirow{5}{*}{0,216} \\
\hline & 1 & 19 & 42,105 & 42,8061 & 0 & 100 & \\
\hline & II & 146 & 57,078 & 44,1104 & 0 & 100 & \\
\hline & III & 128 & 52,865 & 44,5699 & 0 & 100 & \\
\hline & IV & 20 & 33,333 & 41,8854 & 0 & 100 & \\
\hline \multirow{5}{*}{ Salud mental } & 0 & 15 & 70,8667 & 17,45143 & 34 & 96 & \multirow{5}{*}{0,809} \\
\hline & 1 & 19 & 64,4211 & 20,46220 & 5 & 100 & \\
\hline & II & 146 & 68,5342 & 18,27007 & 16 & 100 & \\
\hline & III & 128 & 67,4453 & 20,59654 & 0 & 100 & \\
\hline & IV & 20 & 71,7000 & 17,28431 & 42 & 100 & \\
\hline
\end{tabular}


de trabajo, en la vida social habitual. Y el EC IV (cáncer de mama invasivo) tuvo una fuerte correlación en la dimensión de rol emocional $(0,582)$; presentaron más desánimo, disminución de la valoración de la situación de salud actual y futura.

Al seleccionar a las pacientes con metástasis de los EC II-IV (294), las puntuaciones totales del SF-36 fueron (promedio \pm desviación estándar) para el dolor corporal 57,8 \pm 25,3 , función física $36,1 \pm 40,9$, vitalidad $63,1 \pm 22,6$, y en salud general $60,8 \pm 20,3$. Se observó más afectación en la función física.

Existen diferencias significativas en cada EC cuando se comparan los distintos tipos de tratamientos: tratamiento quimioterápico, hormonal, terapia biológica, quimioterapia, radioterapia, cirugía, cirugía conservadora (lumpectomía) y cirugía radical (prueba ANOVA, p unilateral <0,05) (Tabla VI).

Luego, se hizo el test post hoc Scheffé: hubo diferencias significativas $(p<0,05)$ entre el estadio II comparado con el estadio 0 para el tratamiento con radioterapia, y se realiza más radioterapia en el estadio $\|(0,37)$ versus el estadio $0(0,33)$. Se encontraron diferencias significativas para el estadio I $(0,00)$, estadio $0(0,47)$, estadio IV $(0,80)$ y estadio II $(0,82)$. Respecto al tipo de cirugía, existieron más diferencias significativas para estadio IV $(1,55)$ con mayor tratamiento quirúrgico, comparado con estadio II $(1,01)$ y estadio III $(0,66)$. Para el tratamiento clínico, existieron más diferencias significantes con el estadio IV $(2,45)$ comparado con el estadio III $(1,80)$ y el estadio $0(1,73)$. Este último indica que el estadio con más tratamiento clínico es el IV.

No hubo diferencias significativas para tratamiento hormonal en todos los estadios y para quimioterapia estadio III, porque se halló igual media en los grupos.

Al analizar por ANOVA de una vía para el EC III y dolor, se hallaron diferencias significativas para la presentación del dolor en la mujer con cáncer de mama (p: 0,018). No se encontraron diferencias estadísticamente significativas para los estadios 0, I, II y IV.

Al realizar análisis de correlación de Pearson entre edad y dolor, se encontró una correlación débil negativa para todos los estadios; se especifican algunos de ellos: estadio 0 $(r=-0,492)$, estadio IV $(r=-0,264)$ y estadio I $(r=-0,155)$. La lumpectomía o mastectomía parcial y la mastectomía radical o total tuvieron una correlación débil negativa con $(r=-0,050)$ y $(r=-0,018)$, respectivamente.

\section{DISCUSIÓN}

La media de edad de la muestra de estudio fue 52,8 años y con una desviación estándar de 11,7 años, similar al estudio de Enien y cols., en Egipto, que tuvo una media de 50,3 años y 8,5 de desviación estándar ${ }^{1}$. En el estudio de Sat-Muñoz y

Tabla V. Correlaciones de Spearman significativas $(p<0,05)$ por dimensiones en las pacientes con cáncer de mama

\begin{tabular}{|c|c|c|c|c|c|c|c|c|}
\hline & \multicolumn{8}{|c|}{ Dimensiones de la encuesta SF-36 } \\
\hline & $\begin{array}{c}\text { Salud } \\
\text { general }\end{array}$ & $\begin{array}{l}\text { Función } \\
\text { física }\end{array}$ & Rol físico & $\begin{array}{c}\text { Función } \\
\text { social }\end{array}$ & $\begin{array}{c}\text { Dolor } \\
\text { corporal }\end{array}$ & Vitalidad & $\begin{array}{c}\text { Rol } \\
\text { emocional }\end{array}$ & $\begin{array}{l}\text { Salud } \\
\text { mental }\end{array}$ \\
\hline Edad (general) & - & - & - & - & 0,128 & - & - & - \\
\hline Fatiga & 0,121 & 0,277 & 0,268 & 0,256 & 0,229 & 0,227 & 0,138 & 0,229 \\
\hline \multicolumn{9}{|l|}{$\begin{array}{l}\text { Edad (según } \\
\text { estadio) }\end{array}$} \\
\hline 0 & - & - & - & 0,545 & - & - & - & - \\
\hline 1 & - & - & - & - & - & - & - & - \\
\hline II & - & - & - & - & - & - & - & - \\
\hline III & - & - & - & - & - & - & - & - \\
\hline IV & - & - & - & - & - & - & 0,582 & - \\
\hline
\end{tabular}

Tabla VI. Tipos de tratamiento según estadio clínico

\begin{tabular}{|c|c|c|c|c|c|c|c|c|c|}
\hline \multirow{2}{*}{ Estadio } & \multirow{2}{*}{$\mathrm{n}$} & \multirow{2}{*}{ Quimioterapia } & \multirow{2}{*}{ Hormonal } & \multirow{2}{*}{ Radioterapia } & \multirow{2}{*}{ Biológica } & \multirow{2}{*}{$\begin{array}{l}\text { Quimioterapia } \\
\text { y radioterapia }\end{array}$} & \multirow{2}{*}{ Cirugía } & \multicolumn{2}{|c|}{ Tipo de mastectomía } \\
\hline & & & & & & & & Lumpectomía & Radical \\
\hline 0 & 15 & 13 & 5 & 6 & 0 & 7 & 14 & 14 & 0 \\
\hline I & 19 & 10 & 5 & 9 & 0 & 9 & 19 & 19 & 0 \\
\hline II & 146 & 120 & 40 & 54 & 37 & 10 & 120 & 35 & 75 \\
\hline III & 128 & 101 & 18 & 71 & 30 & 99 & 125 & 46 & 79 \\
\hline IV & 20 & 16 & 0 & 16 & 0 & 11 & 9 & 0 & 9 \\
\hline
\end{tabular}


cols., en México, la edad promedio fue $52,23(10,55)$ años, 17 pacientes $(5,4 \%)$ se encontraban en EC I; $94(29,9 \%)$ en EC II; $101(32,2 \%)$ en EC III y 47 (15\%) en EC IV. En el presente estudio, realizado en el INEN, encontramos $15(4,6 \%)$ en EC 0, 19 (5,8\%) en EC I, 146 (44,5\%) en EC II, 128 (39\%) en EC III y $20(6,1 \%)$ en EC IV, y se observa que predomina el cáncer avanzado ${ }^{18}$.

Para Mehmet Ali Eryılmaz y cols., la edad se correlacionó moderadamente con la función física ${ }^{19}$, se encontró una asociación negativa moderada entre la edad y la función física $(r=-0,235 ; p=0,01)$, mientras que hubo una leve asociación positiva entre la edad y la energía vital $(r=0,198 ; p=0,04)$. Para este estudio, se encontró fuerte correlación entre la edad y la función social.

En la presente investigación, se encontró que el 87,8 \% de las pacientes con cáncer de mama tuvieron dolor, superior a lo hallado por Costa y cols. ${ }^{12}$, con el $71,8 \%$ en el hospital de referencia oncológico del noreste de Brasil, realizado entre julio de 2014 y abril de 2015. El dolor crónico en pacientes por cáncer de mama es una condición frecuentemente encontrada y es usual que aparezca postmastectomía $y$, debido su alta prevalencia, está recibiendo un creciente interés para investigarla en los últimos años ${ }^{20}$. En el estudio de Khan y cols., realizado en India, se encontró que de todos los grupos o conglomerado de síntomas el dolor fue el principal síntoma con el 76,8 \%21. En el estudio de Nho y cols., en Corea, se encontró una prevalencia de dolor en el $73 \%$ de los pacientes ${ }^{22}$.

Los resultados muestran un porcentaje de pacientes con dolor del $87,8 \%$ de las pacientes, lo cual es superior a los datos de otros estudios. De manera análoga, difieren de otros estudios en las dimensiones de la calidad de vida, donde se obtienen las puntuaciones más bajas, concretamente en el rol físico, mientras que otros estudios puntúan peor en la dimensión dolor corporal como parecería obvio, sugiriendo que estas pacientes tienen más dolor que en otros estudios, sin embargo, su calidad de vida se limita más en otros dominios diferentes del dolor corporal. Probablemente, las razones de estas diferencias puedan ser un tratamiento menos óptimo de la enfermedad o un tratamiento inoportuno, por lo que se ha observado actualmente en la atención de consulta externa del hospital; estas variables no fueron investigadas en este estudio. Por otro lado, al usar la escala de dolor en consulta externa de pacientes con cáncer de mama para Lima Metropolitana (escala Lima), esta trata de medir la prevalencia de dolor que presentarían en casa las pacientes con cáncer de mama, que usualmente no son preguntadas con detalle en la consulta clínica por los especialistas de mamas y tejidos blandos o mastólogos o especialistas en medicina paliativa.

Los resultados describen claramente 2 grupos de pacientes con cáncer de mama, un grupo con dolor y otro sin dolor, pero notablemente diferentes en el impacto que el dolor tiene en su estado de salud, por ello las puntuaciones más bajas a la encuesta SF-36 fueron aquellas que tuvieron dolor. El dolor, en general, interviene como una entidad separada y específica de comorbilidad en la mayoría de esta población de mujeres, afectando la calidad de vida. Para muchas pacientes, el dolor crónico comenzó inmediatamente después de la cirugía o temprano en el periodo de tratamiento postquirúrgico. Este tipo de inicio es bien reconocido por los cirujanos de cáncer de mama ${ }^{23}$ y se están realizando varios esfuerzos para tratar este efecto persistente. Sin embargo, el dolor crónico que comienza más tarde, en el periodo postquirúrgico después de semanas o meses y que la mayoría de las pacientes presenta en este estudio, puede ser menos probable de ser reconocido y tratado agresivamente. En particular, el dolor en estas pacientes tiende a ser diagnosticado solo después de un largo periodo de dolor crónico en la consulta externa.

El dolor estuvo inversamente relacionado con la calidad de vida. Existen estudios que en la dimensión dolor corporal generan las puntuaciones más bajas, como el de Domínguez Gil y cols. ${ }^{10}$. En nuestro estudio, la puntuación más baja se obtuvo en la dimensión rol físico.

Las pacientes con cáncer de mama metastásico se vieron más afectadas en la función física y tuvieron baja puntuación de SF-36 con 36,1, dolor corporal 57,8, vitalidad 63,1 y en salud general 60,8; pero en el estudio de Ahmed y cols., el dolor tuvo la puntuación más baja con 22,9 , junto con una función física deficiente de 22,9, poca vitalidad 19,4 y mala salud general con $16,9^{24}$.

Una de las comorbilidades más importantes de las pacientes con cáncer de mama es el dolor; este generalmente se correlaciona en forma negativa o inversa para la calidad de vida. En el estudio de Fu y cols. se encuentra con puntuaciones bajas de la calidad de vida, y del mismo modo para los dominios de salud, funcionamiento físico, dolor corporal y vitalidad ${ }^{25}$.

Entre las preguntas utilizadas con el instrumento validado con alfa de Cronbach 0,931, no encontramos artículos para comparar el dolor al movilizar el brazo, dolor al agarrar objetos, dolor en la noche cuando se encuentra en cama, dolor cuando descansa en el día, dolor al cargar objetos, dolor al hacer ejercicios, dolor en el trabajo habitual, dolor en las actividades del hogar y rigidez del brazo al final del día.

Como limitación, no se abordó la relación entre calidad de vida con el ejercicio físico ya que este último influye en las dimensiones de la vitalidad y aspectos sociales ${ }^{26}$. Tampoco se analizó la influencia del dolor y la calidad de vida por tipo de cirugía, por ser en muchas pacientes incapacitante durante 6-9 meses posteriores al tratamiento primario quirúrgico ${ }^{10}$. No se evaluó la influencia de tratamientos específicos como la radioterapia, que está asociada negativamente con la presentación de dolor corporal ${ }^{27}$.

Por los hallazgos encontrados respecto a la elevada prevalencia de dolor y cómo afectaría la calidad de vida en pacientes con cáncer de mama, y por la relevancia clínica de estos resultados, se hace necesario sugerir la posibilidad de introducir medidas para mejorar el tratamiento del dolor o apertura de nuevas líneas de investigación del dolor en casa o en actividades de la vida cotidiana. Se debería fortalecer las políticas de cuidados paliativos más cercanos al dolor que padece la paciente al estar en su domicilio o en su ambiente de trabajo, o como consecuencia de las actividades cotidianas de la vida.

\section{CONCLUSIÓN}

Hubo diferencias estadísticamente significativas entre las medias de la puntuación global de la calidad de vida relacionada con la salud para todos los EC al comparar los grupos 
con dolor y sin dolor. El EC 0 presentó una fuerte correlación con afectación de la función social, mientras que el EC IV tiene una fuerte correlación en la dimensión de rol emocional. Nueve de cada 10 pacientes con cáncer de mama tienen algún tipo de dolor. El grupo etario de mayor frecuencia fue el de 40-50 años; el EC II fue el más frecuente. El tiempo medio de enfermedad fue 43,1 meses, y los días con dolor en el mes fueron 19. Todas las pacientes presentaron afectación en las 8 dimensiones de la calidad de vida y la fatiga está moderadamente relacionada con la función física, rol físico, dolor corporal, vitalidad y salud mental.

\section{BIBLIOGRAFÍA}

1. Enien M, Ibrahim N, Makar W, Darwish D, Gaber M. Health-related quality of life: Impact of surgery and treatment modality in breast cancer. J Cancer Res Ther. 2018;14:957-63.

2. International Agency for Research Cancer. World Health Organization. Peru. Fuente: Globocan 2018.

3. Ministerio de Salud. Declaran de interés nacional la atención integral del cáncer y mejoramiento del acceso a los servicios oncológicos en el Perú y dictan otras medidas. Decreto Supremo n.o 009-2012-SA. Normas legales. El Peruano; 2012. p. 1-17.

4. Ministerio de Salud. Conforman la Comisión Sectorial encargada de implementar el "Plan nacional de atención integral de cáncer y mejoramiento de los servicios oncológicos en el PerúPlan Esperanza", aprobado por Decreto Supremo 009-2012-SA. Normas legales. El Peruano; 2013. p. 1-4.

5. López Castro R. Prevalencia del dolor en enfermos oncológicos. Dolor irruptivo. Med Paliat. 2015;22:2-9.

6. Smith MD, Meredith PJ, Chua SY. The experience of persistent pain and quality of life among women following treatment for breast cancer: An attachment perspective. Psychooncology. 2018;27:2442-9.

7. Wan BA, Pidduck W, Zhang L, Nolen A, Drost L, Yee C, et al. Patient-reported fatigue in breast cancer patients receiving radiation therapy. Breast. 2019;47:10-15.

8. Schiappacasse Cocio G, Soto PG. Terapia multimodal en la fatiga oncológica: Estudio prospectivo, doble ciego, con asignación aleatoria fase 3. Gac Mex Oncol. 2015;14:85-91.

9. Hart PD, Kang M, Weatherby NL, Lee YS, Brinthaupt TM. Evaluation of the Short-Form Health Survey (SF-36) using the Rasch Model. Am J Public Health Res. 2015;3:136-47.

10. Domínguez Gil MR, Acosta Mosquera ME, Méndez Martín I, Maestre Ramos I, Pedrote Ramírez C, Frutos Cantó M. Evaluación de la Calidad de Vida tras el tratamiento primario del cáncer de mama. Index Enferm. 2009;18.

11. Vilagut G, Ferrer M, Rajmil L, Rebollo P, Permanyer-Miralda G, Quintana JM, et al. [The Spanish version of the Short Form 36 Health Survey: a decade of experience and new developments]. Gac Sanit. 2005;19:135-50.

12. Costa W, Monteiro M, Queiroz J, Gonçalves A. Pain and quality of life in breast cancer patients. Clinics. 2017;72:758-63.
13. Villar RR, Fernández SP, Garea CC, Pillado MTS, Barreiro VB, Martín CG. Quality of life and anxiety in women with breast cancer before and after treatment. Rev Lat Am Enfermagem. 2017;25:e2958.

14. Van den Beuken-Van Everdingen MHJ, Hochstenbach LMJ, Joosten EAJ, Tjan-Heijnen VCG, Janssen DJA. Update on prevalence of pain in patients with cancer: systematic review and meta-analysis. J Pain Symptom Manage. 2016;51:1070-90.e9.

15. Ware JE Jr. SF-36 Health Survey Update. En: Maruish M, editor. The use of psychological testing for treatment planning and outcome assessment. Vol. 3. Mahwah, New Jersey: Lawrence Eribaum Associates; 2004. p. 693-718.

16. Smith DJ, Huntington J, Sloan JA. Choosing the "correct" assessment tool. Curr Probl Cancer. 2006;30:272-82.

17. Hawker GA, Mian S, Kendzerska T, French M. Measures of adult pain: Visual Analog Scale for Pain (VAS Pain), Numeric Rating Scale for Pain (NRS Pain), McGill Pain Questionnaire (MPQ), ShortForm McGill Pain Questionnaire (SF-MPQ), Chronic Pain Grade Scale (CPGS), Short Form-36 Bodily Pain Scale (SF-36 BPS), and Measure of Intermittent and Constant Osteoarthritis Pain (ICOAP). Arthritis Care Res (Hoboken). 2011;63 Suppl 11:S240-52.

18. Sat-Muñoz D, Contreras-Hernández I, Balderas-Peña LMA, Hernández-Chávez GA, Solano-Murillo P, Mariscal-Ramírez I, et al. Calidad de vida en mujeres mexicanas con cáncer de mama en diferentes etapas clínicas y su asociación con características socio-demográficas, estados co-mórbidos y características del proceso de atención en el Instituto Mexicano del Seguro Social. Value Health. 2011;14(5 Suppl 1):S133-6.

19. Pereira J, Silva S, Pereira L, Paludetto KGM. The partial or total mastectomy can interfere with quality of life after treatment of breast cancer. J Phlebol Lymphology. 2013;6:11-8.

20. Tait RC, Zoberi K, Ferguson M, Levenhagen K, Luebbert RA, Rowland K, et al. Persistent post-mastectomy pain: risk factors and current approaches to treatment. J Pain. 2018;19:1367-83.

21. Khan F, Ahmad N, Biswas F. Cluster analysis of symptoms of Bangladeshi women with breast cancer. Indian J Palliat Care. 2018;24:397-401.

22. Nho JH, Kim SR, Park MH, Kweon SS. Symptom clusters and quality of life in breast cancer survivors after cancer treatment in a tertiary hospital in Korea. Eur J Cancer Care (Engl). 2018;27:e12919.

23. Burckhardt CS, Jones KD. Effects of chronic widespread pain on the health status and quality of life of women after breast cancer surgery. Health Qual Life Outcomes. 2005;3:30.

24. Ahmed AE, Alharbi AG, Alsadhan MA, Almuzaini AS, Almuzaini $\mathrm{HS}$, Ali YZ, et al. The predictors of poor quality of life in a sample of Saudi women with breast cancer. Breast Cancer (Dove Med Press). 2017;9:51-8.

25. Fu MR, Axelrod D, Guth AA, Cleland CM, Ryan CE, Weaver KR, et al. Comorbidities and quality of life among breast cancer survivors: A prospective study. J Pers Med. 2015;5:229-42.

26. Castro JL de, Pires-Miranda AK, Farias-Martins-Júnior F, AraujoCosta H, Ferreira-Viana KR, Sevilio-de-Oliveira MN, et al. Influências do exercício físico na qualidade de vida em dois grupos de pacientes com câncer de mama. Rev Bras Ciencias do Esporte. 2016;38:107-14.

27. Bajuk L, Reich M. Calidad de vida relacionada con la salud en mujeres uruguayas con cáncer de mama. Fac Psicol Univ Católica Uruguay. 2011;5:1-12. 